\title{
Role of corollary discharge in space constancy
}

\author{
LAWRENCE STARK and BRUCE BRIDGEMAN \\ University of California, Berkeley, California
}

\begin{abstract}
Visual fixation can be maintained in spite of finger pressure on the monocularly viewing eye. We measured the amount of extraocular muscle effort required to counter the eyepress as the secondary deviation of the occluded fellow eye. Using this method, without drugs or neurological lesions, we have shown that corollary discharge (CD) governs perception of position of a luminous point in darkness, that is, an unstructured visual field. CD also controls visuomotor coordination measured with open-loop pointing and the matching of visual and auditory direction in light and in darkness. The incorrectly biased CD is superseded by visual position perception in normal structured environments, a phenomenon we call visual capture of Matin. When the structured visual field is extinguished, leaving only a luminous point, gradual release from visual capture and return to the biased CD direction follows after a delay of about 5 sec.
\end{abstract}

Helmholtz (1867), following up early studies by Descartes (1664/1972), suggested that, to enable a person to distinguish movements of objects in the world from movements of the retinal image due to eye movements, information about eye movements is compared with sensory information about retinal image movements. In this conception, the visual signal from the retina is compared with the signal that controls eye position, called the "effort of will" (Helmholtz, 1867), "Efferenzkopie" (von Holst \& Mittelstaedt, 1950), or "corollary discharge" (CD) (Sperry, 1950). We use the third term in this paper.

\section{Paralysis Experiments}

In one of his arguments supporting this process, Helmholtz called attention to patients with fresh oculomotor pareses who complained that visual images jumped when they attempted to gaze into the field of action of the paretic eye muscle. He suggested that the efferent "effort of will" driving the eye movement resulted in a perception of displacement because the retinal signal does not change in the paretic field. The mismatch between retinal displacement and the outflowing CD signals gave rise to the perception of displacement, and also to errors in pointing with the hand into the paretic field.

Quantitative extensions of the clinically based paralysis observations have been based on artificial

We are pleased to acknowledge partial s: pport from the NCC 2-86 Cooperative Agreement, NASA-Ames Research Center. This work was carried out while B. Bridgeman was on sabbatical leave from the University of California, Santa Cruz, partially supported by NSF Grant BNS79-06858. Thanks are extended to $\mathrm{M}$ Jeannerod and $A$. Hein for inviting the authors to attend their conference on Space Perception in Lyon, September 1980, at which this paper germinated. The authors are associated with the Department of Physiological Optics (L.S., B.B.) and the Department of Engineering Science and Neurology (L.S.) at the University of California, Berkeley, California 94720 . eye paralysis in normal subjects by mechanical (Mach, 1885/1959; Brindley \& Merton, 1960) or pharmacological (Brindley, Goodwin, Kulikowski, \& Leighton, 1975; Kornmuller, 1930; Siebeck, 1954; Stevens, Emerson, Gersstein, Kallos, Neufeld, Nichols, \& Rosenquist, 1976) means. The recent paralysis experiments of Matin, Picoult, Stevens, Edwards, \& MacArthur (1982) are especially valuable in defining the role of corollary discharge, for they used extensive structured visual fields to take account of visual context as well as control signals. Their subjects sat, nearly completely paralyzed by systemic d-tubocurarine, with their heads tilted slightly back while directing their gaze to a set of illuminated points located at eye height in a normally illuminated room. The $C D$ normally coincides with eye position, but the paralysis reduced the gain of the oculomotor system so that attempts to fixate away from primary position resulted in large deviations of the $C D$ from true eye position in the orbit. Perception was completely normal. When the room lights were extinguished, however, the illuminated points seemed to float down to the floor and remain there. With the head tilted forward, perception also appeared normal in the light but the luminous points drifted upward in the dark.

Matin et al. (1982) explained this phenomenon in terms of a suppression of the $C D$ by information from the structured visual field; as long as the room lights were on, retinal information dominated to tell the visual system that the lights were at eye height. We call this "visual capture of Matin" (VCM), distinguished from other examples of visual capture by accurate perception despite constant error in the $C D$ signal. Without a structured visual field, the $C D$ is the only remaining indicator of position of objects in the visual world, and it gradually comes to dominate perception when a background pattern is removed. Matin et al. also measured visual-auditory 
coordination under these conditions, and found a systematic mismatch between localization and visual stimulus position, even though auditory localization ability is not affected by curare.

These results fundamentally redefine the role of $C D$ in perception and coordination between the senses; the $C D$ signal is overridden in the structured visual field of everyday visual perception, but it continues to function in intersensory coordination. It determines perception when structured retinal information about spatial layout is unavailable.

The work of Matin et al. is based on only one of Helmholtz's proofs for the existence of a $C D$, that is, the observations on patients with naturally occurring paralyses or pareses. We have turned our attention to another of Helmholtz's proofs for the role of $C D$ in perception, the fact that a passive press on the open eye leads to illusory motions of the visual world. [A preliminary version of this material was presented at the 1981 ARVO meeting (Bridgeman \& Stark, 1981).]

\section{Eye-Press Experiments}

The first scientific use of the phenomenon of apparent motion accompanying a press on the eye was made by Descartes (1664/1972), who, in anticipation of the $\mathrm{CD}$ theories developed centuries later, attributed the perceived visual motion to an error in the efferent signals to the eyes. Descartes (1664/1972, p. 64) states: "In that case, the parts of the brain whence the nerves came will not be arranged in quite the same way as they would be" (during a normal eye movement). His optical analysis of the press (Figure 1) is reflected by the traditional interpretation of the phenomenon by Helmholtz and his successors. Descartes's realization of the importance of efferent signals in the resulting perception had been lost, however, and will be revived here.

The traditional analysis of the monocular eyepress experiment, from Descartes to the present, has been that motion in the world is seen because the eye moves without a corresponding $C D$. If fixation is maintained, however, the actual behavior of the oculomotor system is just the reverse: As the finger presses harder and harder on the eye, the oculomotor control system produces a stronger and stronger signal to the eye muscles in a successful effort to keep the fovea centered on the point of fixation. It is the corollary to this motor discharge to the eye muscles alone which leads to the perception of motion. The eyepress, therefore, produces corditions similar to, rather than opposite from, those obtained under oculomotor paralysis: there is a change in efferent signal but no change in retinal signal. Furthermore, the $C D$ no longer matches the true position of the eye, so the eyepress can be used to separate the effect of $\mathrm{CD}$ from eye position. With this technique, we have performed experiments analogous to those of

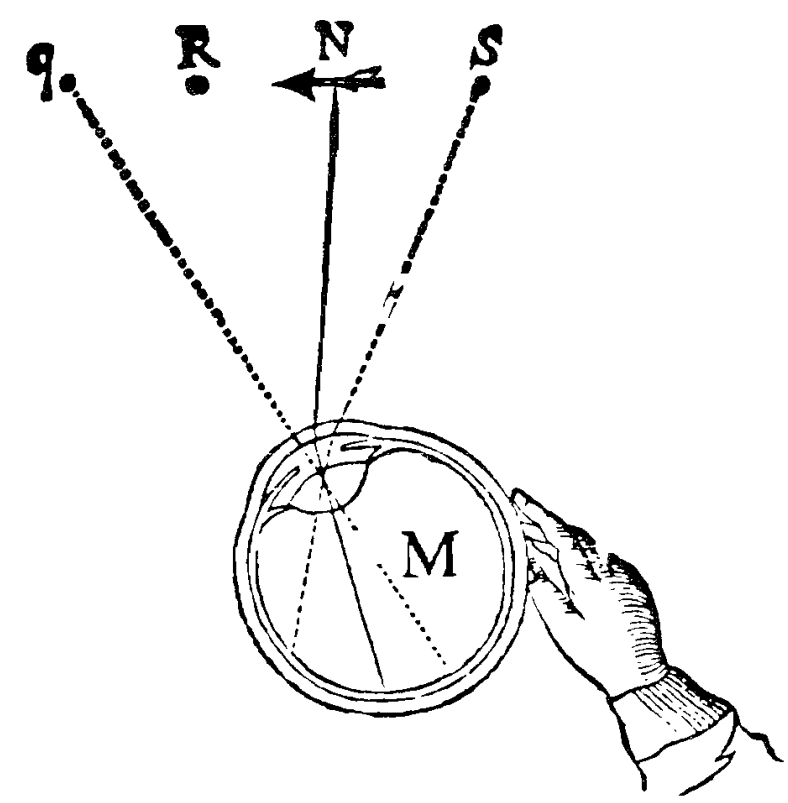

Figure 1. Opdical analysis of the passive eyepress (from Descartes, 1664 (1972). $N$, original fixation point; $q$, fixation point during eyepress. It is assumed that the finger rotates the eye in the head.

Matin et al., appropriately modified, and have performed quantitative experiments on the coordination of vision and pointing.

\section{METHOD}

Experiments were conducted in an apparatus which allowed the subject to see two independently controllable stimuli; one was a structured visual field consisting of a normal laboratory environment rich in carpentered vertical and horizontal surfaces and edges, and the other was a luminous point, $12 \mathrm{arc} \min$ in diameter and viewed through a mirror mounted at a 45 -deg angle to the subject's line of sight, so that the point directly above the mirror appeared to be at eye height and intercepted a line from the viewing eye through the screen parallel to the medium plane (Figure 2). The distance from the screen to the mirror equaled the distance from the mirror to the luminous point, so that small head movements resulted in no deviations of the apparent position of the point on the screen due to parallax. The luminous point appeared to be at the distance of the screen. The optical system was modified from Held and Freedman (1963).

By sighting along the edge of the mirror, the subject could superimpose the luminous point and a pen tip on the screen. $A$ pen mark could then be made on the screen, giving an objective measure of the projection of the luminous point.

For the pointing experiments, a sheet of paper was fastened to the screen and the fixed position of the target point was initially marked with the sighting superimposition method. Then the subject's estimations of the target position, made by marking the screen with a pen held so that the penpoint was as close to the fingertips as possible, could be directly measured on the paper. Pointing was always "open loop," however, because the subject could not see either his hand or the pen when the head was in the chinand-forehead restraint (a very adequate three-point headrest), and he was not told of his accuracy during the experiment. The mirror covered a visual solid angle more than $21 \mathrm{deg}$ wide $\times 28 \mathrm{deg}$ high.

For experiments that required the subjects to adjust the luminous point to the apparent straight-ahead position, the large mirror was replaced by a smaller galvanometer mirror mounted at 


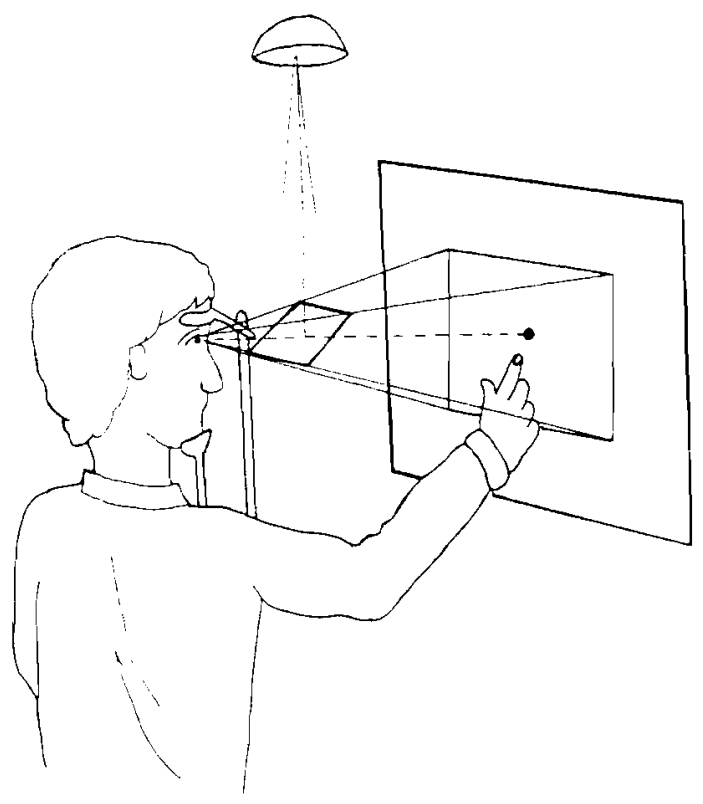

Figure 2. The optical system used for pointing and auditory localization experiments. The mirror hides the screen from the observer, so that open-loop pointing is possible even in lighted environments. Additional baffling (not shown in the figure) aided in blocking visibility of the arm and maintaining open-loop conditions.

the same angle as the front surface mirror, but rotatable about either a vertical or a horizontal axis. All trials with the galvanometer mirror began with the point deviated from the apparent center position, and the subject adjusted the point to appear to be in the straight-ahead position by adjusting a 10-turn potentiometer with a radially symmetric dial, so that the straight-ahead setting could not be inferred from the position of the knob. This arrangement effectively removed proprioceptive arm cues.

Experiments with an acoustic signal used a mechanically generated click repeated at a rate of $4 \mathrm{~Hz}$. Acoustic experiments were carried out only for stimuli moving in the horizontal plane; the width of the vibrating plate of the acoustic stimulus was $22 \mathrm{~mm}$.

The subjects were three adult males with normal or correctedto-normal refraction. ${ }^{3}$

\section{RESULTS}

\section{Experiment 1: The Eye-Press Manipulation}

Because the eyepress has such different origins and results from those traditionally assumed, and because it is the basis for our subsequent experiments, it will be analyzed in some detail.

\section{The Movement is Active, Not Passive}

Under monocular viewing, when pressing on the eye slowly enough for objects to appear to move with moderate speed, there is no difficulty in maintaining fixation on a single visual target. We demonstrated this by asking two of the subjects of our study to perform a forced-choice discrimination, red versus green, before, during, and after the dynamic phase of an eyepress. The targets were square red or green patches, 6 arc min on a side. The colored patch replaced a fixation target for $200 \mathrm{msec}$ before, during, or after an eyepress, and the subject guessed its color. Because of the small target size, this task is difficult enough for performance to be random at $3 \mathrm{deg}$ of retinal eccentricity. Performance was nearly perfect, however, both during the time when the image appeared to move due to an eyepress and during the static holding of the eye in the pressed position. This simple demonstration shows that little or no movement of the target off the fovea occurs during the eyepress maneuver. (Acuity targets could not be used to measure foveal fixation because the eye becomes astigmatic when pressed.)

Lack of image motion on the retina during eyepress is a result of the activity of the saccadic refixation and pursuit tracking systems countering the effects of mechanical pressure on the eye. As analyzed by Bridgeman (1979), it is the CD of the growing oculomotor control signal, rather than the change in retinal image, that leads to the perception of motion during the eyepress.

This active resistance can be demonstrated informally by contrasting the strong resistance to the press by a fixating eye to the negligible resistance by an occluded eye while the fellow eye is fixating. The fixating eye feels stiff to the finger, resisting the pressure with active muscle contraction, while the occluded eye feels flaccid, reflecting only the passive resistance of the orbital tissue and the tonic forces of the extraocular muscles. This effect may also be taken as an indication of the absence of a proprioceptive stretch reflex in extraocular muscles.

\section{Eyepress Yields Translation, \\ Not Rotation of the eye}

When the eye is pressed, its rotation is very small and is directed toward the pressure point. With right monocular viewing, for instance, pressing on the outer canthus of the right eye results in a translation of the eye nasally and a small rotation temporally to maintain position of the image on the translated retina (Figure 3). This can be shown by two methods. First, a subject can do the maneuver, maintaining gaze on a fixation point, while an experimenter measures the deviation of the pupil with a ruler. This measure is quick and direct, but inaccurate; there. fore, a second, more accurate but subjective, method was employed. Translation was measured accurately by mounting a reference point halfway between the subject's eye and the tangent screen and noting the apparent projection of the point on the screen during the press compared with its position without a press. With the head restrained, the difference in projections onto the tangent screen equals the translation of the eye in the orbit. This value ranged from 3 to $6 \mathrm{~mm}$ in our subjects. 
Translation of the eye, in the absence of errors in fixation of a target $50 \mathrm{~cm}$ from the eye, results in a compensatory rotation of the eye in the direction opposite the translation, but of small magnitude (Figure 3). For our subjects, this counterrotation was 0.3 to $0.6 \mathrm{deg}$ in magnitude, resulting in no change in the position of the retinal image of the tangent screen. When the eye is translated, less sclera is visible on the side of the eye opposite the press. Because eye position in another person is usually judged by the amounts of sclera visible on the sides of the iris, it is easy for an observer to misattribute the translation of the eye to a passive rotation in the direction of the press. Without fingerpress, the eye does not normally translate within the orbit, either by theory (Fry \& Hill, 1962, estimated that 1/200th of a 10deg rotation might be translation due to a nonstationary center of rotation) or by experimental measurement (Krishnan \& Stark, 1977, measured a 15arc-min-equivalent maximum bound for such movements).

This analysis applies to monocular viewing with the eyepress only. For binocular conditions, the subject's response is more complicated, for Hering's

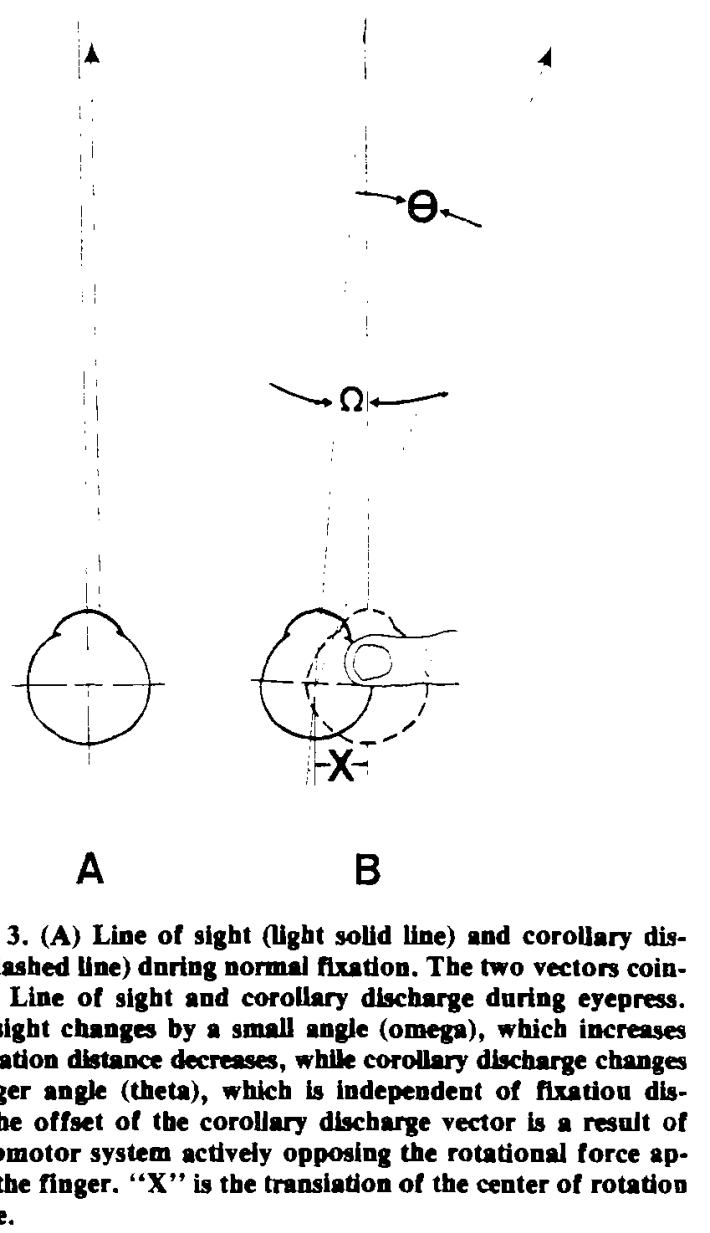

$(1868 / 1977)$ law dictates that the same innervation directs both eyes. When one eye is artificially deviated, however, no innervation that can direct both eyes to the same location in space exists, and diplopia often results. A subject has several possible strategies in this situation, yielding an ambiguity in interpretation of the attempted goals of the innervations and their results. Therefore, all viewing in our experiments was monocular, sighting through the pressed eye.

It was necessary for our subjects to practice the eyepress maneuver extensively before beginning experimental sessions. A moderate press on the eye resulted in a deviation that increased with the pressure on the eye but reached a saturation after maximum translation of the eye had been achieved. At higher pressures, retinal ischemia set in more quickly and little additional apparent deviation of the visual world resulted. Horizontal and vertical apparent deviations could be generated independently with practice, the deviations tending to be horizontal when the head was tilted forward and the eye was pressed while looking at a target at eye height; tilting the head backward and aiming the eye slightly temporally resulted in a primarily vertical deviation. The best vertical deviations were obtained by pressing not on the outer canthus of the eye, but on the lower eyelid, using it as a cushion to press the cornea directly upward. With practice, the subjects could generate highly reproducible apparent target shifts and could consistently initiate the required ocular conditions by pressing the eye in complete darkness. That the eyepress was reproducible is evidenced by the fact that the variance in eyepress clearly cannot exceed the measured variance of the experimental results (Table 1).

\section{Secondary Deviation as a Measure of Coroilary Discharge}

During an eyepress, the occluded eye is free to move, even though the viewing eye does not rotate significantly when fixated on a target. In the fixating eye, rotation induced by the eyepress is balanced by rotational effort of the extraocular muscles, so that the retinal image does not move. Because the occluded eye is not restricted in its motion, this rotational effort should result in a secondary deviation of the occluded eye, because Hering's law requires corresponding muscles of the two eyes to receive the same innervation. Eye position and $C D$ will continue to match in the unencumbered occluded eye. Therefore, to obtain an objective measure of the difference between corollary discharge and position of the pressed eye, we measured secondary deviations in the occluded eye during presses on the other eye.

Horizontal eye movements were measured with an infrared photocell system (Stark, Vossius, \& Young, 1962) which offers high spatial and temporal resolu- 
tion without contacting the eye. Following calibration, eye movements were recorded continuously while the eye was pressed and released horizontally in the manner of the above experiments.

Figure 4 shows objective records of the secondary deviation of the occluded eye and the inferred CD of the pressed eye. Its dynamics show a mixture of saccades and smooth pursuit to compensate for the effect of the eyepress, a movement pattern that cannot be generated voluntarily in the absence of a moving target. However, our experiments are static and do not examine the relationship of CD dynamics and oculomotor dynamics; final eye position could generate $C D$ as well as the dynamic phase of saccades and smooth pursuit. Due to a slight unsteadiness of the eye pressure, drift of the signal during the press is greater than that during normal fixation in the same subject. The magnitude of the drift, however, is much smaller than the offset effect. The records shown here demonstrate a deviation ranging from

\section{Trial}

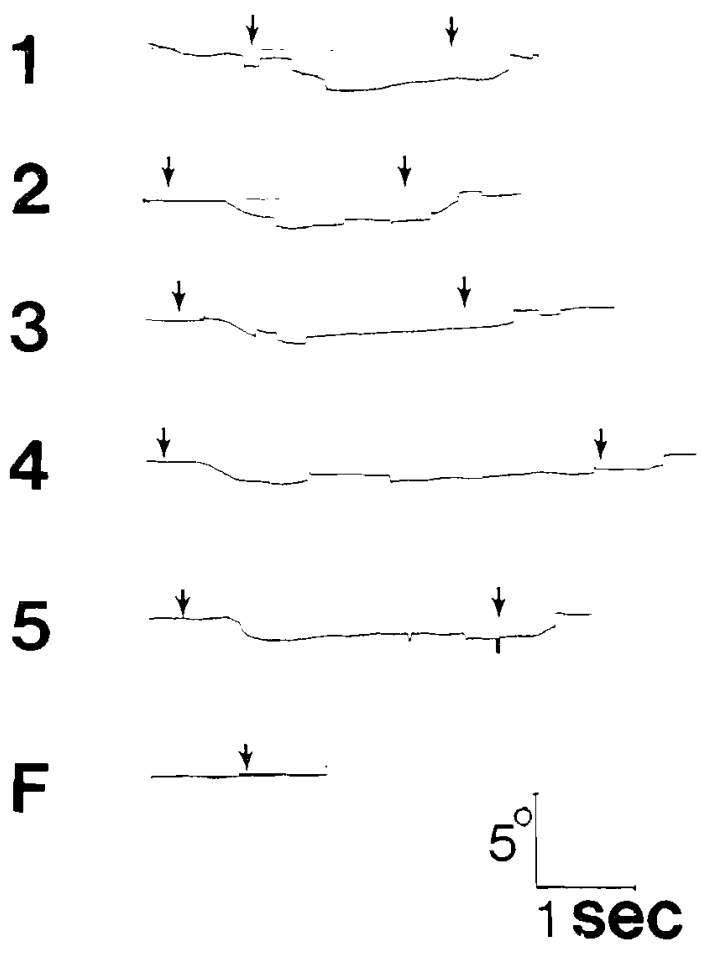

Figure 4. Secondary deviations of an oceluded left eye during pressure on the exposed right eye (Subject B.B.) Recordings were made with an infrared system which does not contact the eye or require the use of visible light for recording. Five consecutive trials of eyepress (first arrow in each record) and release (second arrow) are shown. Latencies between the arrows and the eye movements include the reaction time from the "press" or "reiesse" commands by the experimenter to the finger movement. $F$ is a control interval of fixation without eyepress. Note the presence of normal microsaccudes in the occluded eye, even during the eyepress. (Left $=$ up.)
1.25 to $2.2 \mathrm{deg}$, falling within the range of the deviations obtained in Experiment 2 and 3 below.

Another experiment (not illustrated) showed that when the eye was pressed in complete darkness, there was no concomitant motion of the fellow eye. Thus, extraretinal eye position information is corollary to the motor command, and therefore, we can drop the imprecise expression "extraretinal signal."

\section{Discussion}

Absence of secondary deviations under open-loop conditions without a target demonstrates that only outflow influences eye position. If proprioceptive inflow from muscle spindles, tendon organs, or other mechanoreceptors were able to influence the oculomotor control system in these conditions, then the inflow should generate a secondary deviation of the occluded eye (in contradiction to Skavenski, 1972). Because the observed deviations match exactly the requirements of the outflow conception, the terms "outflow," "extraretinal signal," and "corollary discharge" are considered interchangeable in this paper.

CD may not always be an exact "Efferenzkopie"; in prism experiments, adaptation to the bias may be produced by an adaptation changing the quantitative relationship between the $C D$ and the efferent (oculomotor) signal. In this case, the $C D$ is a corollary but not a copy. Prism adaptation, however, may also modify the afferent signal or the quantitative comparison between the afferent signal and the $C D$.

The inaccuracy of the CD led MacKay (1973) to propose that the $C D$ might provide only a qualitative indication that image movement should be expected, so that the expectation would be resolved by a retinal signal from the movement itself. By clarifying the distinction between $C D$ and saccadic suppression; we show that $C D$ is, indeed, adequate to participate in quantitative constancy so that the Mackay suggestion becomes unnecessary.

In discussing his qualitative theory of the corollary discharge, MacKay (1973) uses the eyepress maneuver to show the sensory results of moving the entire retinal image passively across the retina. The results of Experiment 1 show, however, that only outflow causes movement perception in this case. In a further experiment with important implications for space constancy, MacKay describes a condition in which a stroboscopically illuminated field is seen during an eyepress. The absence of apparent motion of the stroboscopic field implied to MacKay that the visual system is less sensitive to displacements than to continuous motions, although our results show that the apparent motions seen during the eyepress are uniquely related to $C D$ and therefore to the space constancy mechanism itself. Because the stroboscopic field is a poor stimulus for pursuit eye tracking, the pursuit 
movements that would cause a deviation of the CD might not occur in this case, so that changes in $C D$ could not contribute to a perception of motion.

Our reanalysis of the eyepress maneuver shows that eye pressure can dissociate the $C D$ from the eye position itself. Ordinarily, the two coincide, but the effort of the oculomotor system to compensate for the eyepress results in a deviation of the $C D$ from the actual orientation of the eye. The dissociation creates conditions adequate to test results previously obtained from patients with eye muscle paresis or with subjects under a partial or complete paralysis.

The eyepress offers several theoretical and practical advantages, however. First, it is performed in normal undrugged subjects, so that its generalizability is more secure. Second, each subject can serve as his own control. Because the condition is quickly and completely reversible, experimental and control trials can be interlaced. Third, both motor activity and the other senses are left intact, so that we can measure the coordination of vision with the motor system during the dissociation of retinal information and $C D$. This combination of advantages is unique to the eyepress experiments, with the two alternative methods of investigating dissociation of extraretinal and retinal signals being the paralysis experiments and the experiments on patients with paralyzed eye muscles.

\section{Experiment 2: Judgment of Visual Direction}

\section{Qualitative Judgment}

Unstructured field. When a subject pushed on the eye while only the luminous point was visible, the point underwent a rapid and permanent deviation in space. The apparent dynamics of the point's deviation matched the dynamics of the eyepress itself. Although adjusted to appear straight ahead in normal lighting, the point continued to appear offset from straight ahead as long as an eyepress was maintained. The magnitude of the effect was estimated by mounting a scale on the tangent screen and turning off all lights to remove the structured visual field after the subject had become familiar with the distances indicated on the scale. Then the luminous point was turned on. Estimated deviations were $2-2.5 \mathrm{~cm}$ horizontal and 5-6 cm vertical for Subject B.B. and 6.5$7 \mathrm{~cm}$ horizontal and $5-6 \mathrm{~cm}$ vertical for Subject W.Z. These deviations occurred in spite of identical retinal conditions in both cases. The subjects expressed a lack of confidence in the accuracy of their estimates, although these deviations are comparable to the deviations found below with other methods.

Structured field. In a normally structured laboratory environment, each subject first ascertained what object was directly in front of his eyes. The structured field was then extinguished, the subject pressed on his eye to yield either a vertical or a horizontal deviation, and the room was reilluminated.
The subject then made another judgment about which object was directly in front of his eye. Again, retinal stimulus conditions were identical in both cases. Under these conditions, the world continued to look completely normal. Except for an astigmatism caused by the geometric effects of the finger pressure, there was no sensory indication of abnormality, and objects that had appeared to be directly in front of the observer in normal conditions continued to look that way during the static press. These effects were observed for all three subjects.

\section{Visual Axis, Method of Adjustment}

In the dark condition, the subject began by pressing the eye in complete darkness. The target point was then illuminated. The subject next adjusted the galvanometer mirror until the point appeared to be directly ahead-either at eye height, if the adjustment was vertical, or intersecting the plane through the center of rotation of the eye and parallel to the median plane, if the rotation was horizontal. After adjustment, the subject released his eye and marked the position of his judgment on the screen, using the superimposition method. Control trials, interspersed with the experimental trials, were identical, except that the eye was not pressed.

The eyepress resulted in a consistent error in the straight-ahead setting in both vertical and horizontal phases of the experiment. The deviation was statistically significant for all subjects (Table 1 ), and is shown in Figure 5 (top) for the subject who had the median magnitude of offset effect pooled across conditions. Thus, when a purely visual test is used, and other modalities are not involved, the deviation in the corollary discharge caused by the eyepress has a clear effect in darkness without visual context. The horizontal conditions replicate the excellent experiment of Skavenski, Haddad, and Steinman (1972).

Still sitting in the galvanometer mirror apparatus with the head restrained at chin and forehead, the subject in the light condition set the luminous point to be directly in front of the exposed eye in the context of the structured visual field. All illumination was extinguished, the subject pressed the eye in darkness, and both the luminous point and the structured field were illuminated together while the subject maintained the eye pressure after the experimenter had offset the mirror left or right at random. The subject adjusted the galvanometer mirror while holding the eye until the point appeared to be directly in front of the eye.

Experimental settings of the galvanometer mirror were not significantly different from control settings in either vertical or horizontal conditions (Table 1), showing that the of fset of the cofollary discharge had no effect in the presence of a structured visual field.

When the eye was pressed in the light and the structured field was then extinguished while the subject maintained the pressure, the target point seemed to 
Table 1

Experimental Results

\begin{tabular}{|c|c|c|c|c|c|c|c|c|}
\hline \multirow[b]{3}{*}{ Experiment } & & & \multicolumn{6}{|c|}{ Subject } \\
\hline & & & \multicolumn{2}{|c|}{ B.B. } & \multicolumn{2}{|c|}{ W.Z. } & \multicolumn{2}{|c|}{ L.S. } \\
\hline & & & Dark & Light & Dark & Light & Dark & Light \\
\hline 2: Visual Axis & $\mathrm{HZ}$ & $\begin{array}{l}\text { Mean } \\
t(d f) \\
p\end{array}$ & $\begin{array}{l}1.9 \\
3.88(34) \\
.001^{*}\end{array}$ & $\begin{array}{l}0.1 \\
0.46(10) \\
.67\end{array}$ & $\begin{array}{l}1.9 \\
6.86(30) \\
.001^{*}\end{array}$ & $\begin{array}{l}1.5 \\
1.17(8) \\
.28\end{array}$ & & \\
\hline \multirow{3}{*}{ 3: Pointing } & VT & $\begin{array}{l}\text { Mean } \\
t(d f) \\
p\end{array}$ & $\begin{array}{l}5.1 \\
6.93(8) \\
.001^{*}\end{array}$ & $\begin{array}{l}0.4 \\
1.75(10) \\
.11\end{array}$ & $\begin{array}{l}3.3 \\
6.55(20) \\
.001^{*}\end{array}$ & $\begin{array}{l}0.2 \\
0.09(8) \\
.94\end{array}$ & & \\
\hline & $\mathrm{HZ}$ & $\begin{array}{l}\text { Mean } \\
t(d f) \\
p\end{array}$ & $\begin{array}{l}3.5 \\
8.41(9) \\
.001^{*}\end{array}$ & $\begin{array}{l}2.3 \\
14.41(6) \\
.001^{*}\end{array}$ & $\begin{array}{l}1.7 \\
5.51(8) \\
.001^{*}\end{array}$ & $\begin{array}{l}2.0 \\
6.66(10) \\
.001^{*}\end{array}$ & $\begin{array}{l}2.9 \\
5.29(8) \\
.001^{*}\end{array}$ & $\begin{array}{l}5.5 \\
9.06(12) \\
.001^{*}\end{array}$ \\
\hline & VT & $\begin{array}{l}\text { Mean } \\
t(d f) \\
p\end{array}$ & $\begin{array}{l}2.5 \\
5.70(10) \\
.001^{*}\end{array}$ & $\begin{array}{l}2.0 \\
4.03(8) \\
.001^{*}\end{array}$ & $\begin{array}{l}2.1 \\
2.92(8) \\
.001^{*}\end{array}$ & $\begin{array}{l}1.0 \\
1.58(11) \\
.142\end{array}$ & $\begin{array}{l}7.2 \\
7.11(8) \\
.001^{*}\end{array}$ & $\begin{array}{l}3.8 \\
4.60(8) \\
.0018 *\end{array}$ \\
\hline 3: Acoustic Matching & $\mathrm{HZ}$ & $\begin{array}{l}\text { Mean } \\
t(d f) \\
p\end{array}$ & $\begin{array}{l}2.7 \\
3.43(18) \\
.003^{*}\end{array}$ & $\begin{array}{l}1.9 \\
1.44(18) \\
.167\end{array}$ & $\begin{array}{l}2.3 \\
2.32(18) \\
.032\end{array}$ & $\begin{array}{l}1.9 \\
1.95(8) \\
.067\end{array}$ & $\begin{array}{l}6.5 \\
3.17(17) \\
.0056^{*}\end{array}$ & $\begin{array}{l}2.2 \\
3.66(8) \\
.0064^{*}\end{array}$ \\
\hline
\end{tabular}

Note-Three quantitative tests for spatial localization for three subjects. Dark refers to unstrictured, and light to structured visual fields. Horizontal (HZ), but not vertical (VT), deviations were obtained for acoustic matching. Mean differences in degrees between eyepress and non-eyepress (means) ore given together with $t$ values and associated degrees of freedom (df) that yield the probability (p). $\quad * p<.01$.

remain stable for several seconds before beginning a slow drift in the direction of the deviated corollary discharge. The drift was difficult to estimate quantitatively, but it seemed to begin after about $7 \mathrm{sec}$ for Subject B.B., 4-5 sec for L.S., and 1-2 sec for W.Z. The direction of motion generally was not exactly vertical or horizontal, and the target reached its resting asymptotic position after about $15 \mathrm{sec}$ from lightoff. The resulting deviation had a sigmoid character, with an initial stability, a period of drift, and a final stable phase in the fully deviated position. There was never an abrupt jump or abrupt acceleration of the target.

\section{Visual Capture}

The lack of change of perceived straight-ahead in a structured visual field during the eyepress is an example of visual capture of Matin (VCM), and is parallel to Matin's observations of visual capture in perception during paralysis. Our subjects continued to observe distinct transient movements of the entire visual world during the phasic components of the eyepress, however, despite successful fixation on a single object. Because only the CD signal is changing at this time, this suggests that the transient components of the $C D$ can influence perception in the light even though its static values do not. It is as if the CD affected perception through a high-pass filter.

The release from VCM provides a first look at the information-storage capabilities of the cognitively based system which judges visual position on the basis of cues from the structure of the visual field.
Information derived from this source is perfectly preserved for several seconds after it ceases to be available due to extinguishing of the visual context. For the first few seconds of darkness, the system continues to suppress the CD and rely on the temporally extrapolated visual context information. When the $\mathrm{CD}$ finally begins to influence position perception, its signal is gradually combined with the extrapolated context information in such a way that no sharp acceleration or abrupt movement of the luminous point is perceived. Rather, the perception results from a weighted combination of the two sources of information, with the corollary discharge gradually coming to dominate the perceived position in darkness. Ordinarily, the switchover from one source of information to the other is unnoticeable, because both sources register the same position.

This pattern of behavior may reflect the visual system's attempt to optimize the quality of the information available to it. When two sources are in conflict, the less precise source, the $C D$, is rejected in favor of the visual context information. The system continues to trust this information more than the $C D$ for several seconds after it ceases to be available. But, since the organism and/or objects in the world normally are in motion, this temporal extrapolation gradually ceases to be more trustworthy than the alternative internal signal. During the next seconds, there is never a double localization from the two conflicting sources of position information: rather, an integrator gradually replaces the inflow visual context estimate of position with the outflow $C D$ esti- 


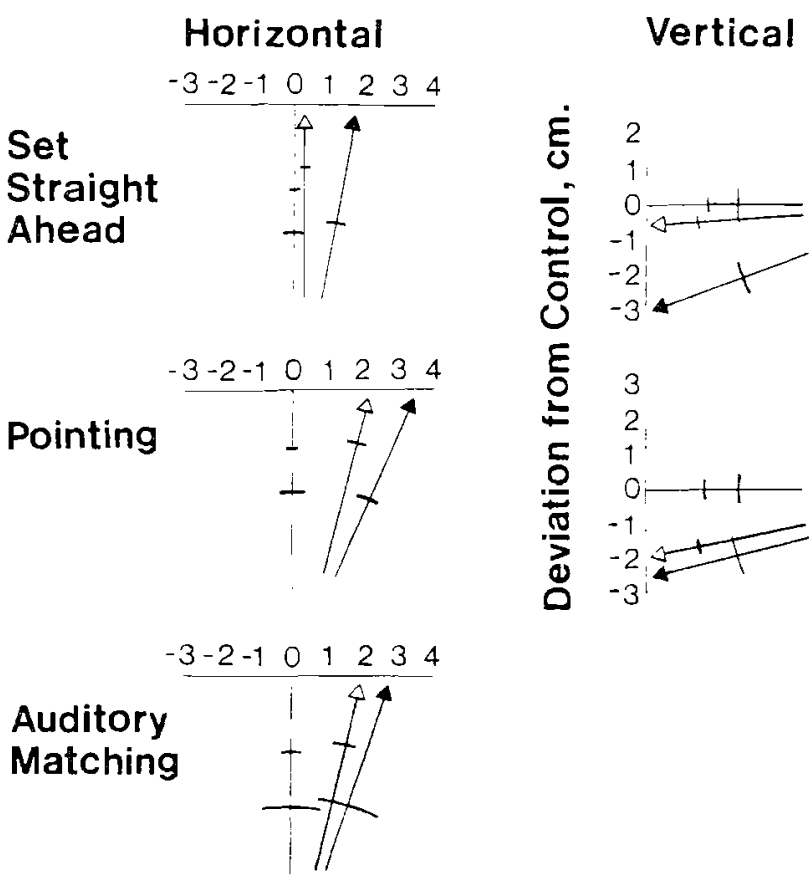

Flgure 5. Position determinations in light (open arrows) and dark (filled arrows) for Subject B.B. Offets from the center are in degrees, with negative degrees representing left or down. Solid arcs on each arrow represent \pm 1 S.D. for each condition. The control values are always represented on the straight-ahead axis. Top row: Judgments of visual straight ahead (Experiment 2), showing settings of the galvanic mirror. Middle row: Open-loop pointing with a fully extended arm (Experiment 3), using the fixed mirror and using the superimposition method of Figure 2. Bottom row: Positions of the audltory stimulus when the subject determined it to be superimposed with the light (Experiment 3). Only horizontal measurements were made in this experiment. The horizontal "set straight ahead" arrow, light condition, was nearly superimposed on the control axis and is represented parallel to it.

mate. During this period, the perception matches neither signal. The perception parallels the fact that the extrapolated visual signal does not lose its information value abruptly, but only gradually becomes less trustworthy than the $C D$. This observation contrasts with that of Bischof and Kramer (1968), who sometimes observed double localization of targets flashed during saccades in darkness.

In contrast, when a light comes on in complete darkness, no information other than corollary discharge is ever available, and the system immediately uses the CD signal alone to estimate position.

\section{Experiment 3: Interaction With Other Modalities}

\section{Pointing to Apparent Target Position}

In the absence of a structured visual field, the subject pressed on the eye in complete darkness and then asked the experimenter to illuminate the target point. He then marked the screen at the place where the target light seemed to intersect it. Experimental and control trials were interspersed.
Pointing bias in the dark for all subjects (Table 1) showed a consistent deviation in the direction of the offset CD. When the right side of the right eye was pressed to the left, the pointing was biased to the right; when the cornea was pressed upward, the pointing deviated downward.

Pointing following the extinguishing of the background lights was biased immediately, and the bias remained constant in the absence of a structured visual field. Figure 6 shows pointing estimates of target position following background light offset, with two trials at each delay. There was one pointing trial at a variable delay following each light offset. Estimated position of the light shows no relation to the time spent in darkness: the slope of the best-fit linear regression line is -0.038 . This result contrasts with the estimates of perceived direction following light offset: pointing remains stably offset from the true position, even while the perceived position of the point is undergoing a drift.

In the light condition, both the target point and the structured visual field were continuously visible. The subject pressed on the eye and marked the screen with a pen held by the fingertips so that the penpoint would be superimposed with the luminous point's apparent position on the screen. Again, marks made during eyepressing were alternated with marks made in the normal control condition without pressing. Despite the presence of visual context, open-loop pointing was significantly biased, in both vertical and horizontal directions (Figure 5, middle). Pointing was consistently offset despite the lack of an impression of abnormality in perceptual conditions during pressing. The pointing bias coexisted with visual capture in perceptual judgment without apparent conflict, even during the pointing sessions.

A trial-by-trial matching of the deviation of pointing with deviation of the $C D$ signal (Experiment 1;

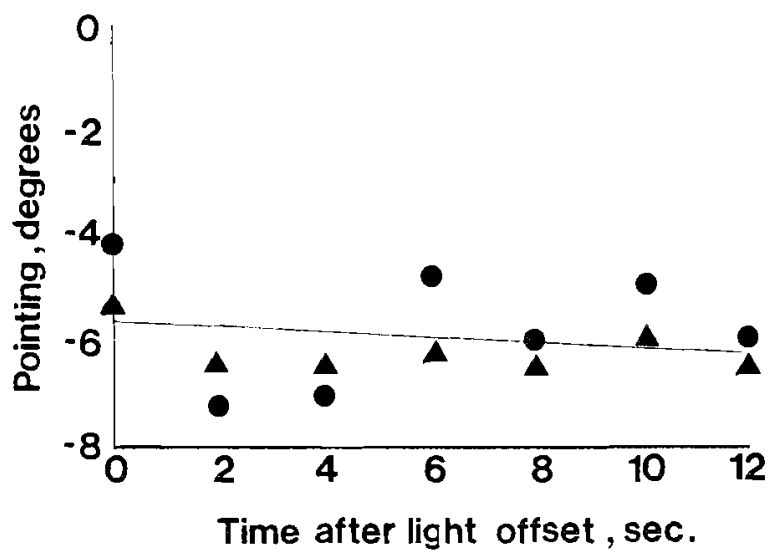

Figure 6. Absence of dynamical changes in pointing to the tangent screen after light offset (Experiment 3). During vertical eyepress, polnting was consistently below the ohjective light position (at 0 deg). Triangles and filled circles, two trials on consecutive days, Suhject B.B. 
Figure 4) was not possible because most of the variance in pointing originated in the motor system, not in the retinal input or $C D$. This result caused us to examine the variance in pointing to determine whether it was greater in the experimental condition than in the control condition with normal fixation. The ratio of the variances was compared with an $F$ test for each subject. In two of the subjects, there was no significant difference in the variances (for L.S., $F=1.14$, $p=.38$; for W.Z., $F=1.13, p=.39$ ), while, in the third subject, a higher variance in the experimental condition was marginally significant (B.B., $F=2.43$, $\mathrm{p}=.03$ ). If pointing variance is not reliably greater in the eyepress condition, the additional variance in pointing added by variations in the eyepress is not significant.

\section{Matching of Acoustic and Visual Direction}

The experimenter moved the sound source directly in front of the screen laterally at a rate of about $2 \mathrm{deg} / \mathrm{sec}$. The subject controlled the motion of the auditory stimulus verbally, with the commands "'move left," "move right," and "stop," until he was satisfied that the sound source was superimposed on the luminous point. The experimenter followed these commands with eyes closed to avoid bias in moving the sound source. When the subject was satisfied that the sound source was superimposed with the light, the experimenter marked the position with a pen fixed to the sound source and performed another trial, beginning with auditory stimulus motion from the opposite side of the center line. The subject could not see the sound source in this condition. The procedure was the same in darkness, with only the luminous point visible, and in the structured visual field.

The results of the acoustic matching were similar to the pointing results, although the acoustic match yielded higher variances and therefore lower statistical significance levels; perhaps some of this increased variance is due to the $22-\mathrm{mm}$ width of the vibrating plate of the acoustic stimulus.

As expected, auditory offset was similar with and without the presence of a structured visual field (Figure 5 , bottom). The offsets of pointing in Figure 5 are slightly greater in darkness in all conditions for the subject illustrated. This was not always true in either of the other subjects, however, and a test of mean light vs. dark estimation (pointing and auditory) pooled across subjects and conditions shows no significant differences $[\mathrm{t}(10)=0.523$, n.s.]

\section{Discussion}

Corollary discharge is robust, having effects on coordination of vision with other modalities in both structured and unstructured visual fields. The similarity of average offset with acoustic and pointing measures shows that visual capture does not affect either visuomotor or intersensory integration, the deviated CD signal dominating both measures. This result confirms the results obtained by Matin et al. (1982) in paralysis experiments.

In addition, we have shown that the visuomotor coordination, measured by open-loop pointing, is biased by the CD in both structured and unstructured fields, to the same extent as the other measures of the bias. Objective determinations of the change in the $C D$ during the eyepress, obtained by measuring secondary deviations in the occluded eye (Experiment 1), are in agreement with the magnitudes of the biases found in Experiments 2 and 3.

All of these results are consistent with classical corollary discharge theory, except for visual capture, which requires a modification of the theory to assume that $C D$ is superseded in perception when visual context provides higher quality spatial information.

\section{GENERAL DISCUSSION}

Using the eyepress method to dissociate eye position from $C D$, we have shown that the bias of $C D$ leads to consistent of fsets of perceptual position estimates and intersensory integrations in unstructured fields, evidence for its continued presence. Only in the presence of a structured visual field does VCM prevent the biased $C D$ from influencing perceptual judgments.

\section{Cognitive and Ambient Systems}

Conditions under which the corollary discharge influences behavior can be compared with the twovisual-systems dichotomy (Schneider, 1968, 1969; Trevarthen, 1968), which has gained wide acceptance. A "focal" or cognitive system is cortically based and governs pattern perception, recognition, and memory, while an "ambient" motor-oriented system handles the coordination of vision with space. The two systems have independent topographic representations of space in normal humans because each representation can be biased without affecting the other (Bridgeman, Kirch, \& Sperling, 1981; Bridgeman, Lewis, Heit, \& Nagle, 1979). Their interactions can be surprisingly subtle (Gregory, 1958; Paillard, 1980).

To a first approximation, we can generalize that $C D$ is the source of ambient system information, but $C D$ enters the cognitive system only when a structured visual field is absent. CD governs pointing, for instance, even when it contradicts VCM. The major exception is the phasic influence of $C D$ on perception: the discharge provides unambiguous information about the time and direction of change in position, even though its tonic component is superseded in a structured visual field. When structure is not available, the discharge provides both tonic and phasic information to perception. 


\section{Space Constancy}

Our experiments also contribute to a consistent reinterpretation of space constancy, the apparent stability of the visual world despite saccades, with and without a structured visual field. In terms of the proximal stimulus, the classic definition of the problem is that displacements of images on the retina lead to perceptions of displacement during visual fixation, while the same displacements induced by saccadic eye movements yield no perception of displacement.

Helmholtz showed the importance of CD in space constancy by citing examples of apparent movement of the retinal image elicited without displacement on the retina, that is, during paralysis. Our results support this view in two ways: First, a transient perception of movement of the world intrudes into perception during a dynamic eyepress with constant visual fixation (even though VCM eventually supercedes the offset $C D$ in perception of the visual. straightahead in structured environments). Second, CD is present and influences behavior in structured visual fields when measured by pointing or auditory localization. An additional factor increases the apparent accuracy of CD. During saccades, the saccadic suppression of image displacement (Bridgeman, Hendry, \& Stark, 1975; Mack, 1970; Stark, Kong, Schwartz, Hendry, \& Bridgeman, 1976) prevents perception of small displacements of visual images that are introduced during saccadic eye movements.

\section{REFERENCES}

Bischor, N., \& Kramer, B. Untersuchungen und Überlegungen zur Richtungswahrnehmung bei willkürlichen sakkadischen Augenbewegungen. Psychologische Forschung, 1968, 32, 195-218.

Bridgeman, B. Adaptation and the two-visual systems hypothesis. Behavioral and Brain Sciences, 1979, 2, 84-85.

Bridgeman, B., Hendry, D., \& Stark, L. Failure to detect displacement of the visual world during saccadic eye movements. Vision Research, 1975, 15, 719-722.

Bridgeman, B., Kirch, M., \& Sperling, A. Segregation of cognitive and motor aspects of visual function using induced motion. Perception \& Psychophysics, 1981, 29, 336-342.

Bridgeman, B., Lewis, S., Heit, G., \& Nagle, M. Relation between cognitive and motor-oriented systems of visual position perception. Journal of Experimental Psychology: Human Perception and Performance, 1979, 5, 692-700.

Bridgeman, B., \& Stark, L. Efferent copy and visual direction. Investigative Ophthalmology and Visual Science Suppl., 1981, $20,55$.

Brindley, G., Goodwin, G., Kulikowski, J., \& Leiohton, D. Stability of vision with a paralysed eye. Journal of Physiology (London), 1975, 253, 65p-66p.

Brindley, G., \& Merton, P. The absence of position sense in the human eye. Journal of Physiology (London), 1960, 153, $127 \cdot 130$.

Descartes, R. Treatise of man (T. S. Hall, trans. \& Ed.). Cambridge, Mass: Harvard University Press, 1972. (Originally published 1664.)

FRY, G. A., \& HiLl, W. W. The center of rotation of the eye. American Journal of Optometry \& Archives of the American Academy of Optometry, 1962, 39, 581-595.
GREGORY, R. Eye movements and the stability of the visual world. Nature, 1958, 182, 1214-1216.

HELD, R., \& FREEDMAN, S. Plasticity in human sensorimotor control. Science, 1963, 142, 455-462.

Helmholtz, H. von Handbuch der physiologischen Optik. Leipzig: Voss, 1867.

HERING, E. The theory of binocular vision (B. Bridgeman \& L. Stark, Eds., \& B. Bridgeman, trans.). New. York: Plenum Press, 1977. (Originally published 1868 .)

Holst, E. von, \& Mittelstaedt, H. Das Reafferenzprinzip: Wechselwirkungen zwischen Zentralnervensystem und Peripherie. Naturwissenschaften, 1950, 37, 464-476.

Kornmuller, A. Eine experimentelle Anesthesie der ausseren Augenmuskeln am Menschen under ihre Auswirkungen. Journal für Psychologie und Neurologie, 1930, 41, 354-366.

Krishnan, V. V., \& Stark, L. A heuristic model for the human vergence eye movement system. IEEE Transactions on Biomedical Engineering, 1977, BME-24, 44-49.

MACH, E. Analysis of sensations (C. M. Williams, trans.). New York: Dover, 1959. (Originally published 1885.)

MACK, A. An investigation of the relationship between eye and retinal image movement in the perception of movement. Perception \& Psychophysics, 1970, 8, 291-298.

MACKAY, D. Visual stahility and voluntary eye movements. In R. Jung (Ed.), Central processing of visual information (Part $A$, Vol. V111/3), New York: Springer Verlag, 1973.

Matin, L., Picoult, E., Stevens, J., Edwards, M., \& MacArthur, R. Oculoparalytic illusion: Visual-field dependent spatial mislocalizations by humans partially paralyzed with curare. Science, 1982, 216, 198-201.

Paillard, J. The contribution of peripheral and central vision to visually guided reaching. In D. Engle, M. Goodale, \& R. Mansfield (Eds.), Advances in the analysis of visual behav. ior. Cambridge, Mass: M.I.T. Press, 1980.

SChNEIDER, G. Contrasting visuomotor functions of tectum and cortex in golden hamster. Psychologische Forschung, 1968, 31, 52-63.

SCHNE IDER, G. Two visual systems. Science, 1969, 163, 895-902.

StEBECR, $\mathbf{R}$. Wahrnehmungsstoerung und Stoerungswahrnehmung bei Augenmuskellaehmungen. Von Graefes Archiv für Ophthalmologie, 1954, 155, 26-34.

SKavensk 1, A. A. Inflow as a source of extraretinal eye position information. Vision Research, 1972, 12, 221-229.

Skavenski, A. A., Haddad, G., \& Steinman, R. M. The extraretinal signal for the visual perception of direction. Perception \& Psychophysics, 1972, 11, 287-290.

SPERRY, R. Neural basis of spontaneous optokinetic response produced by visual inversion. Journal of Comparative and Physiological Psychology, 1950, 43, 482-489.

Stark, L., Kong, R., Schwartz, S., Hendry, D., \& Bridoeman, B. Saccadic suppression of image displacement. Vision $R e$ search, 1976, 16, 1185.1187.

Stark, L., Vossius, G., \& Young, L. Predictive control of eye tracking movements. IRE Transactions on Human Factors in Electronics, 1962, HFE-3, 52-57.

Stevens, J., Emerson, R., Gersstein, L., Kallos, T., Neufeld, G., Nichols, G., \& Rosenquist, A. Paralysis of the awake human: Visual perceptions. Vision Research, 1976, 16, 93-98.

Trevarthen, C. Two mechanisms of vision in primates. Psychologische Forschung, 1968, 31, 299-337.

\section{NOTE}

1. L.S., emmetropic presbyope; W.Z., emmetrope; and B.B., intermittent exotrope.

(Manuscript received September 2, 1982; revision accepted for publication July 11,1983 .) 